|| ISSN(online): 2589-8698 || ISSN(print): 2589-868X ||

International Journal of Medical and Biomedical Studies Available Online at www.ijmbs.info

NLM (National Library of Medicine ID: 101738825)

Index Copernicus Value 2020: 79.44

Case Report.

Volume 6, Issue 02; February: 2022; Page No. 06-11

\title{
MANAGEMENT OF ODONTOMA ASSOCIATED WITH UNERUPTED TOOTH: CASE REPORT
}

\author{
Muhammad Adityo Imam Nugroho ${ }^{1}$, Winarno Priyanto ${ }^{2}$, Endang Sjamsudin ${ }^{3}$ \\ ${ }^{1}$ Resident department of Oral and Maxillofacial Surgery Faculty of Dentistry University of Padjadjaran, Dr. Hasan \\ Sadikin Hospital, Bandung, Indonesia
}

${ }^{2}$ Staff of Oral and Maxillofacial Surgery Department, Dr. Hasan Sadikin Hospital, Bandung, Indonesia ${ }^{3}$ Staff of The Department of Oral and Maxillofacial Surgery, Faculty of Dentistry, University of Padjadjaran, Bandung, Indonesia

Article Info: Received 03 December 2021; Accepted 20 January 2022

DOI: https://doi.org/10.32553/ijmbs.v6i2.2411

Corresponding author: Muhammad Adityo Imam Nugroho

Conflict of interest: No conflict of interest.

\section{Abstract}

Introduction: Odontomas represent the most common type of odontogenic benign jaws tumors among patients younger than 20 years of age. There are two types of odontoma called compound odontoma and complex odontoma. There is no difference in treatment between compound and complex odontomas, however, it needs consideration in treatment so that trauma is minimized and does not cause effects. The aim of this case report is to describe a minimally invasive surgical procedure to remove a complex odontoma localized in the mandible area associated with an unerupted permanent third molar.

Case Report: A case of a 16 years old female came with complained of pain and swelling in the impacted tooth area since 5 months ago with swelling in the unerupted tooth area. Radiographically, there was a lesion located over the impacted tooth a complex odontoma-associated of impacted mandible molar is presented. The patient was diagnosed with an odontoma complex Methods: The method used in this case is performed with minimally invasive surgical technique is adopted to remove the least amount of bone tissue as far as possible. The result of treatment in this case found a mass of $4 \mathrm{~mm}$ in size accompanied by 38 tooth. The prognosis in these patients is ad bonam

Discussion: The purpose of this technique is to preserve as much as possible the surrounding bone tissue in order to promote healing and cause less discomfort to the patient during postoperative time. In this case, it was found that the lesion was located above the unerupted tooth, which is usually in the case of odontoma the lesion was under the unerupted tooth. This technique was chosen because the mass can be removed and the unerupted tooth is removed so that the source of the infection will heal completely without causing recurrence but in this case there is a risk of paresthesia because it is close to the inferior alveolar nerve.

Conclusion: The treatment of choice for complex odontomas is surgical removal, followed by histopathological analysis to confirm the diagnosis. Complications such as fractures root, loose second molar, trauma to the temporo-mandibular joint, mandible fractures and paresthesia must be considered. Adequate knowledge of clinical characteristics is necessary to establish proper diagnosis and management of odontomas.

Keywords: Odontoma, Complex Odontoma, Lesion, Odontogenic Tumor

\section{Introduction}

Odontoma is an odontogenic, non-aggressive benign tumor and is a hamartomatous developmental disorder. Odontoma develops from primordial odontogenic tissue. The composition is a combination of odontogenic epithelium and odontogenic ectomesenkhim. The term odontoma was first proposed by Paul Broca in 1867. Such tumors account for approximately $22 \%$ of all odontogenic tumors and are most often diagnosed in thesecond decade of life and the most common location is the anterior region of maxilla. Usually these tumors are not symptomatic, and the most common causes are permanent dental impaction and persistence of the first-born teeth.[1,2]
According to the 2005 World Health Organization classification, odontoma is an odontogenic benign tumor of the young age[2]. Despite this, odontomas are clinically considered as tumor like formations (hamartomas of dental tissues) or developmental anomalies, rather than true odontogenic neoplasms[3]. Two main types of odontoma are described: (a) complex odontoma, an amorphous and disorderly pattern of calcified dental tissues, and (b) compound odontoma, multiple miniature or rudimentary teeth [4-5]. The compound odontoma has predilection toward the anterior maxilla (61\%), whereas only $34 \%$ of complex odontomas occur in this area; the complex type shows a predilection for the posterior jaws (59\%) and lastly 
the premolar area $(7 \%)$. Both variants are made of all dental tissues such as enamel, dentin, cementum, and pulp. [6,7] Etiology of these tumors is unknown, nevertheless, some authors have described the possible relationship with trauma in primary dentition. Malassez paradental remains, inflammation processes, odontoblastic hyperactivity and heredity anomalies (Gardner and Herman syndrome).[8-9] It has been suggested that local factors, such as trauma and infection, can facilitate conditions for its appearance. Odontomas may also be associated with failure of primary tooth eruption, missing permanent teeth, dentigerous cyst formation, and, rarely, calcifying odontogenic cysts.[10]

Sixty percent of complex odontoma occurs in women and found in the second decade of age, whereas odontoma compound generally occurs in adolescence [11]. Although compound odontoma and complex odontoma in its development do not cause pain, odontoma can cause maxillary bone deformity or mandibles that experience such odontoma[3,5]. Odontoma is usually barn accidentally known from the radiological picture due to the presence of teeth that do not grow, or the presence of pain in the area of teeth that do not grow[5]. Handling odontoma is an operative mass extirpation of odontoma accompanied by the retrieval of teeth that do not grow due to the presence of odontoma [11]. Treatment of choice consists in conservative surgical enucleation by means of the removal of the conjunctive tissue capsule that surrounds it, suitably preparing the sample for the histopathological study which will support an accurate diagnosis. Sometimes, orthodontic treatment will be required to reposition the arch of the retained tooth. Prognosis is generally favorable, with scarce relapse index; relapserate increases when enucleation is conducted during the first calcification stage [11].
The purpose of this technique is to preserve as much as possible the surrounding bone tissue in order to promote healing and cause less discomfort to the patient during postoperative time. In this case, it was found that the lesion was located above the unerupted tooth, which is usually in the case of odontoma the lesion was under the unerupted tooth. This technique was chosen because the mass can be removed and the unerupted tooth is removed so that the source of the infection will heal completely without causing recurrence but in this case there is a risk of paresthesia because it is close to the inferior alveolar nerve.

\section{Case Report}

A 16-year-old female patient came up with a complaint of swelling at left lower jaw region since 5 months ago. A week later, the patient complained of pain and swelling around the absence teeth. The patient went to the dentist in Banjaran area and was given antibiotic and anti inflamatory drugs. Two months ago patient complained of the swelling getting bigger, the patient came to the hospital in the Soreang area with visible swelling of the left cheek region and was performed a panoramic $\mathrm{x}$-ray with result it was seen that there was an unerupted tooth with a lesion on it. The patient was referred to Hasan Sadikin Hospital and had not history reported oral trauma or infection. History of drug and food allergies was denied. Intraoral examination showed the presence of the primary left lower jaw with a diagnosis of odontoma complex at 38 region with impaction of tooth 37 and cemento-ossifying fibroma were considered for differential diagnosis. An excisional biopsy was performed in 38 regions with odontectomy of tooth 37 . The prognosis in this case is ad bonam.
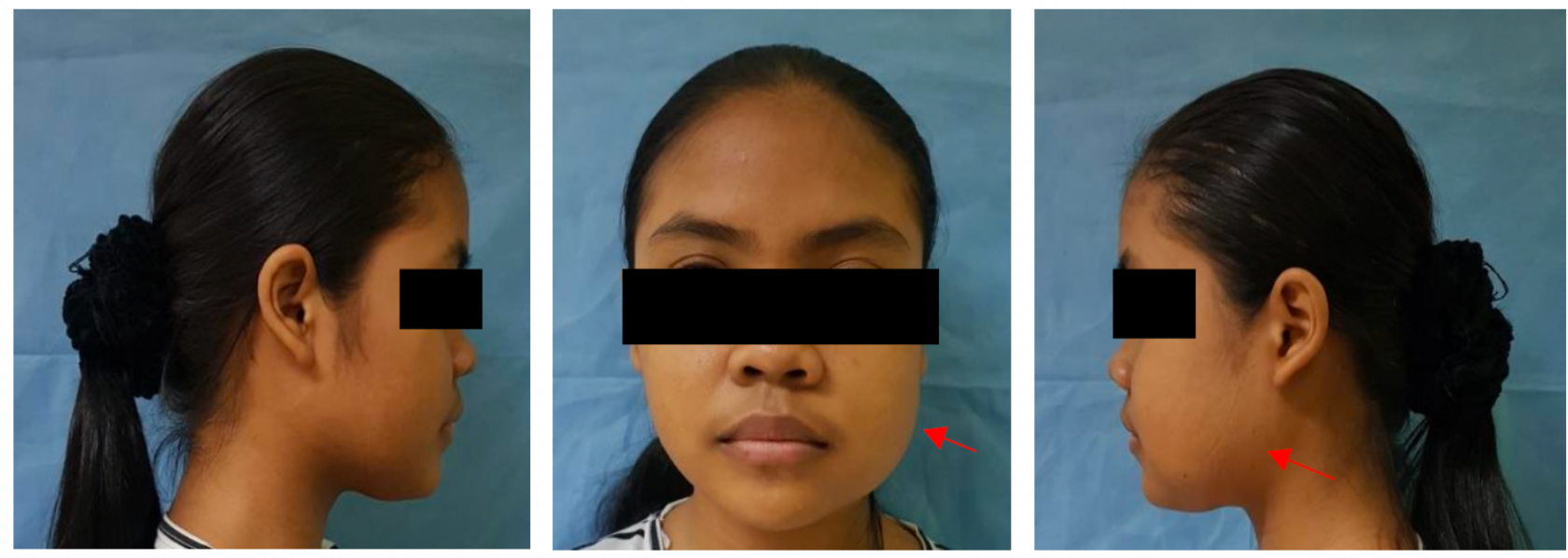

Figure 1: Clinical examination there was extraoral swelling on the left side of face. 

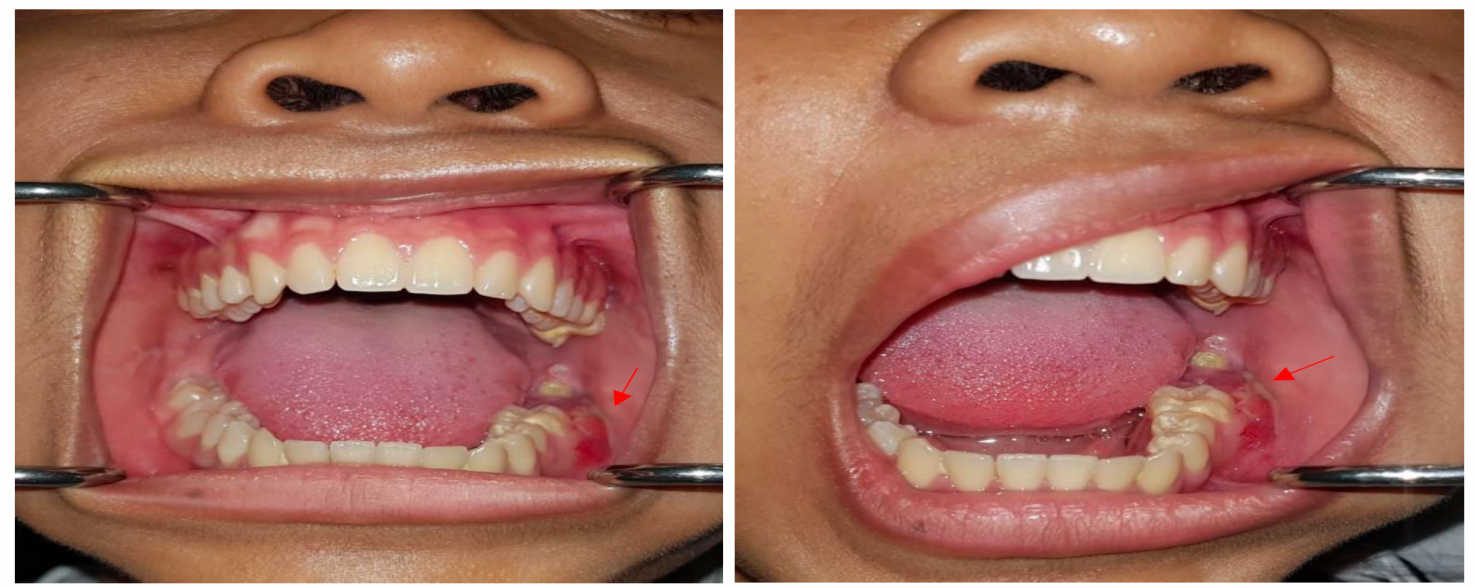

Figure 2: Intraoral examination showed there was a red lesion around the unerupted tooth

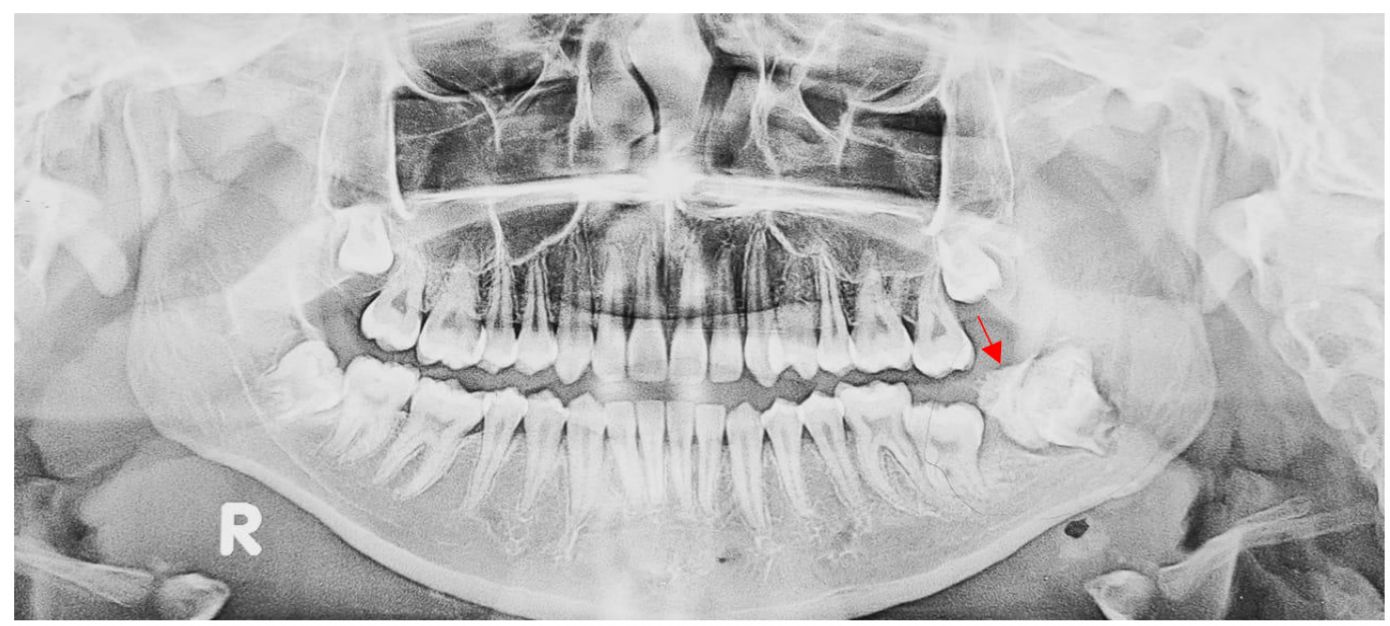

Figure 3: Panoramic X-ray

A radiographic examination (panoramic X-ray) showed multiple radiopaque structures compatible with a provisional diagnosis of complex odontoma and the unerupted left lower jaw (Figure 3).
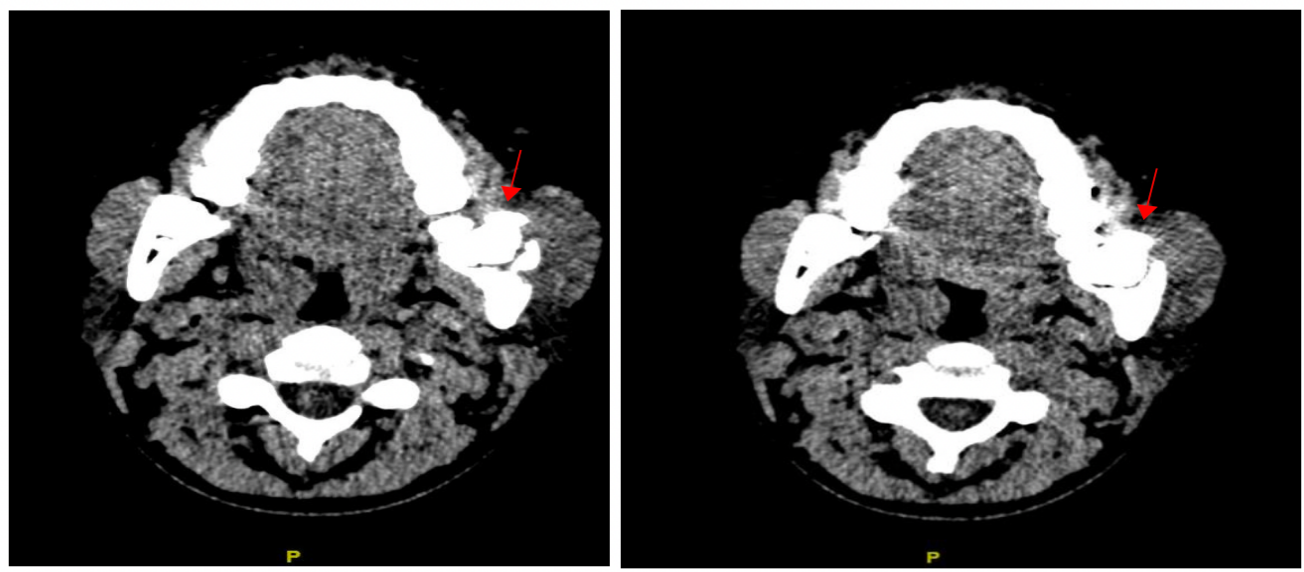

Figure 4: 
A computed tomography (CT) with was performed in order to define mass demarcated with septated irregular edges, not accompanied by cortical left mandibular ramus suggestive of odontoma, enlarged left submandibular lymph node, no intracranial metastases were seen (Figures 4)

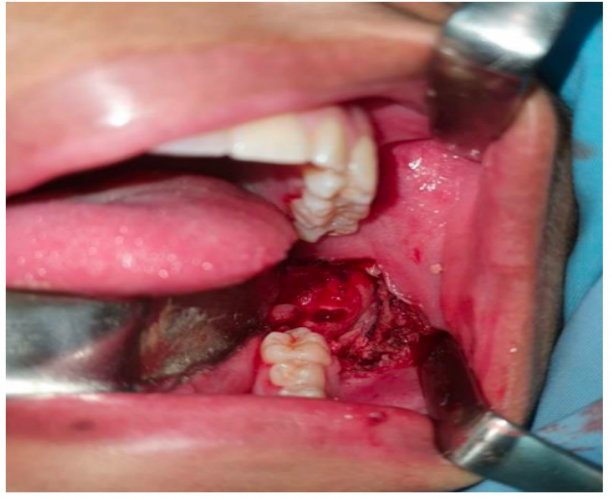

$\mathbf{a}$

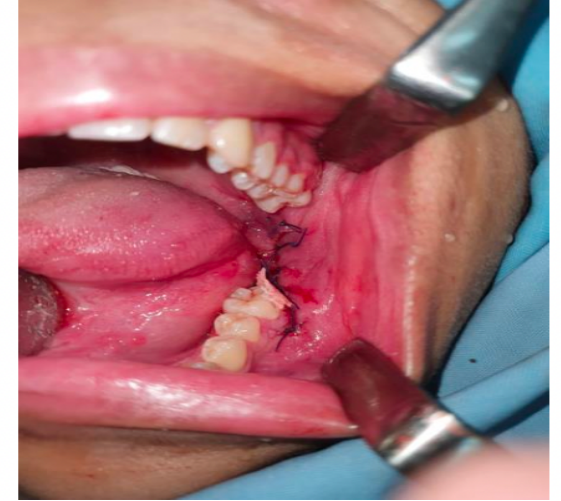

b

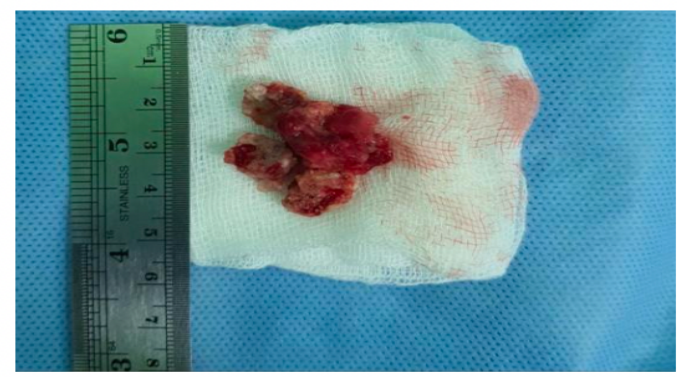

c.

Figure 5:

(a) Intraoral left buccal vestibule incision and elevation of buccal and lingual mucoperiosteal flap. (b) Iodoform gauze application and suturing to the operation area. (c) Removal of odontoma and removal of tooth 38

\section{Discussion}

Although complex odontoma usually presents as an asymptomatic lesion, it may be sometimes associated with one or more pathological changes. In the present case, the patient presented with mild facial asymmetry, a small extraoral swelling. Radiographic appearance of complex odontoma is determined by developmental stage and degree of mineralization. In the present case, the lesion was in third stage, which is described as a completely radioopaque lesion (masses of dental tissues) with a radiolucent surrounding zone (connective tissue capsule.. [21]

Odontomas are the most common type of benignodontogenic tumors of the oral cavity [17]. They are considered a malformation (hamartomas) instead of a neoplasm. According to the WHO, it is a congenital defect in development, resulting in the growth of epithelial and mesenchymal cells fully differentiated tissues in dental[18]. In meta-analysis was possible to observe theoccurrence of odontomas of which $61.3 \%$ are composedof odontomas and $37 \%$ complex. As for location, most lesions appear in the maxilla (56\%) and mandible (44\%). Odontomas has no predilection for gender and can occur at any age. Other studies point to a higher incidence in the first two decades of life[19]. Odontomas are usually asymptomatic, but can cause problems in the eruption of primary teeth and the impaction or eruption of permanent teeth delayed[20,21]. The complex form of odontomas is less common than the compound type and is more often associated with unerupted teeth. The results of our study are consistent with previous research, and we observed a clear predominance of compound lesions over complex odontomas.[22] Presently, the World Health Organization (WHO) classifies odontoma within the category of odontogenic tumors (OT) composed by epithelium and odontogenic ectomesenchyme with or without formation of mineralized dental tissue[1,15,23]. There are two classifications: compound (CpO) and complex (CO)[22]. In relation to tumor location, the majority of the compound odontomas are found in the anterior maxilla while the most common location of complex odontomas is the posterior mandible followed by 
the anterior maxilla.[22] Compound odontogenic tumor (CpO) exhibits morphological and histological differentiation, while complex odontogenic tumor (CO) only presents histological differentiation. In $\mathrm{CpO}$ multiple amorphous dental structures are formed (denticles), while in CO a solid mass of dental soft and hard tissues is formed, these tissues are haphazardly arranged and do not resemble the morphology of a tooth.[16]

The treatment of choice is generally surgical removal to prevent associated complications. Clinical and radiographic follow-up should be performed when surgical treatment is deferred [22]. In general, the management of odontomas is almost the same, lesions in odontoma of small size is performed surgery excision and usually no recurrent lesions, whereas in large odontoma lesions, excision can affect bone and tooth tissue around it. 9 Odontoma case this time, big lesion involving the mandibular first molar, so that the extraction was carried out on the tooth[24]. Complications of Odontoma are the tooth shape of this odontoma is like normal teeth, tends to erupt but is inactive, if it is partially erupted, caries will quickly develop. If not treated quickly, this caries will develop rapidly and can infect soft tissues and cause neuralgia. Adequate knowledge of clinical characteristics is necessary to establish proper diagnosis and management of odontomas. The present case of complex odontoma is unique because of its association with agenesis of permanent mandibular second molar.[24]

\section{Conclusion}

Mandible multiple complex odontomas constitute a therapeutic challenge. Since in some cases, lesions are located nearby vital anatomic structures. In this case, to prevent the injury of the mental nerve, after the detachment and lesion recognition. Odontoma is a benign neoplasm that usually does not cause pain. However, pain can occur due to infection of the area around the odontoma. In addition, odontoma can cause neurological pain when pressing on the nerves below. Odontoma treatment is done by excisional and removal of the teeth. Thus, we readily identified this structure during all surgeries, decreasing the postsurgical risks.

\section{References}

1. S.-Y. An, C.-H. An, and K.-S. Choi, "Odontoma: a retrospective study of 73 cases," Imaging Science in Dentistry, vol. 42, no. 2, pp. 77-81, 2012.

2. S. Vaid, R. Ram, V.K. Bhardwai, M. Chandel, P. Jhingta, N. Negiand D. Sharma. Multiple compound odontomas in mandible:A rarity. Contemp Clin Dent. 2013; 3(3):341-343.

3. B. L. Nelson and L. D. R. Thompson, "Compound odontoma," Head and Neck Pathology, vol. 4, no. 4, pp. 290-291, 2011.
4. P. Boffano, E. Zavattero, F. Roccia, and C. Gallesio, "Complex and compound odontomas," Journal of Craniofacial Surgery, vol. 23, no. 3, pp. 685-688, 2012.

5. Iatrou, E. Vardas, N. Theologie-Lygidakis, and M. Leventis, "A retrospective analysis of the characteristics, treatment and follow-up of 26 odontomas in Greek children," Journal of Oral Science, vol. 52, no. 3, pp. 439-447, 2010.

6. G. Yildirim-Oz, G. Tosun, D. Kiziloglu, E. Durmu s, and Y. Sener, "An unusual association of odontomas with primary teeth," European Journal of Dentistry, vol. 1, pp. 45-49, 2009.

7. Hidalgo O, Leco MI, Martinez JM. Metaanalysis of the epidemiology and clinical manifestations of odontomas. Med Oral Patol Oral Cir Bucal. 2012; 13 (11): 730-734.

8. Iatrou I, Vardas E, Theologie-Lygidakis N, Leventis M. A retrospective analysis of the characteristics, treatment and follow-up of 26 odontomas in Greek children. J Oral Sci. 2011; 52 (3): 439-447.

9. Chrcanovic BR, Jaeger F, Freire-Maia B. Two-stage surgical removal of large odontoma complex. Oral Maxillofac Surg DOI 10.1007/s 10006-01000206-0.

10. Regezi JA, Sciunbba JJ. Oral Pathology Clinical Pathologic Correlation. Philadelphia: Saunder Co; 1989.p.363-4.

11. Manoj V. Large erupting odontoma complex: A case report. J Can Dent Assoc 2007; 73: 169-72.

12. Altay A M, Cehreli C Z, Ozgur B. Management of a Compound Odontoma in the Primary Dentition. Journal of denstistry for Children, 2016.

13. Agarawal R, Himani G, Mandeeep S, J Vaibhav. A Large Complex Odontome Associated with Impacted 2nd Molar Displacing the Mandibular Canal: A Rare Case Report. International Journal of Applied Dental Science, 2016; 2(2): 86-89.

14. Nascimento MM, Almeida CM, Nonaka CF, Odontoma Associated with Impacted Mandibular Canina: Surgical Guided Eruption. DOI 10. 1590/1981- 863720160002000113001.

15. Barba LT, Campos DM, Rascon MN, Barrera VR. Descriptive Aspects of Odontoma: Literature Review. Vol. 20, No. 4, pp. e256-e269, 2016.

16. R.S. Baldawa, K.C. Khante, J.V. Kalburg, V.O, Kasat. Ortho-dontic management of an impacted maxillary incisor due to odontoma. Contemp Clin Dent. 2011; 2(1): 37-40.

17. Santos DM, Gois AS, Amorim KS, Cavalcanti RL, Junior A. Compound Odontoma Associated to Permanent Teeth Impacted in Jaw: Case Report. Journal of Oral Diagnosis. 2018; 03:e20180031.

18. K.S. Kannan, R. Prabhakar, R. Saravanan, Karthikeyan, Ra- jvikram. Composite Compound Odontoma-A Case Report. JClin Diagn Res. 2013; 7(10): 2406-2407. 
19. N. Raval, D. Mehta, K. Vachhrajani and A. Nimavat. Erupted odontoma: a case report. J Clin Diagn Res. 2014; 8(7):ZD10-1.

20. Bereket C, Cakir-Ozkan N, Bulut E, Tek M. Complex and compound Odontomas: Analysis of 69 Cases and a Rare Case of Erupted Compound Odontoma. Nigerian Journal of Clinical Practice. 2015. Vol. 18.

21. Rana V, Srivastava N, Kaushik N, Sharma V, Panthri P, Niranjan MM. Compound Odontome: A Case Report. International Journal of Clinical Pediatric Dentistry. Vol. 12, 2019.
22. Tantanapornkul W, Kaomongkolgit R, tohnak S. Erupted Compound Odontoma Associated with Missing Tooth: A Rare Case Report. Journal of Dentistry Indonesia 2020, Vol. 27, No. 1, 46-49

23. Angelo C, Grechi M, Garg AK. Surgical Treatment of Impacted Tooth Associated with a Big Odontoma: Piezosurgery Approach. Acta Scientific Dental Science 4.5 (2020): 48-51.

24. Misra SR. Complex Composite Odontoma: A Cause of Impacted Teeth. Indian Journal of ForensicMedicine and Toxicology, 2020, Vol. 14, No. 4. 